\title{
An unusual presentation of post gastric bypass hypoglycemia with both postprandial and fasting hypoglycemia
}

\author{
Xin Chen', Dina Kamel2, Braden Barnett2, Evan Yung³, Adrienne Quinn ${ }^{4}$ and \\ Caroline Nguyen²
}

1Division of Internal Medicine, LAC+USC Medical Center, University of Southern California, Los Angeles, California, USA, ${ }^{2}$ Division of Endocrinology, Diabetes, and Metabolism, Keck School of Medicine, LAC+USC Medical Center, University of Southern California, Los Angeles, California, USA, 3Division of Pathology, LAC+USC Medical Center, University of Southern California, Los Angeles, California, USA, and ${ }^{4}$ Keck School of Medicine, University of Southern California, Los Angeles, California, USA

Correspondence should be addressed to $X$ Chen

Email

xchen5@dhs.lacounty.gov

\section{Summary}

There has been an increasing awareness of post gastric bypass hypoglycemia (PGBH). Histopathologic findings from such patients who underwent partial/total pancreatomy, however, can vary widely from minimal changes to classic nesidioblastosis, making the pathologic diagnosis challenging. PGBH typically presents as postprandial hypoglycemia, as opposed to insulinoma, which presents as fasting hypoglycemia. Herein, we describe an unusual case of a patient with PGBH who initially presented with postprandial hypoglycemia three years after surgery, but later developed fasting hyperinsulinemic hypoglycemia as the disease progressed. Our hypothesis for this phenomenon is that this disease is progressive, and later in its course, the insulin release becomes dissociated from food stimulation and is increased at baseline. Future studies are needed to investigate the prevalence as well as etiology of this progression from postprandial to fasting hypoglycemia.

\section{Learning points:}

- There has been an increasing awareness of post gastric bypass hypoglycemia (PGBH).

- Histopathologically, PGBH can vary from minimal changes to nesidioblastosis.

- Although uncommon, patients with PGBH after Roux-en-Y gastric bypass may present with both postprandial and fasting hyperinsulinemic hypoglycemia as disease progresses.

- Our hypothesis for this phenomenon is that the insulin release becomes dissociated from food stimulation and is increased at baseline with disease progression.

\section{Background}

Treatment of obesity with bariatric surgery has gained increasing popularity because of demonstrated effectiveness in decreasing obesity-related comorbidities. Prevalence of severe hypoglycemia after Roux-en-Y gastric bypass (RYGB), marked by neuroglycopenic symptoms, is estimated to be between 0.2 and $0.36 \%$, based on a large cohort study $(1,2)$. PGBH tends to occur between 1 and 8 years post procedure and presents as postprandial hypoglycemia. Fasting hypoglycemia is a very rare manifestation of PGBH. The prevalence of fasting hypoglycemia has not been well established in the population with PGBH except for a few case reports in the past two decades (3). Herein, we describe an unusual case of PGBH who initially presented with 
postprandial hypoglycemia and later developed fasting hyperinsulinemic hypoglycemia as disease progressed.

\section{Case presentation}

A 34-year-old woman with a past medical history of RYGB as treatment for obesity, complicated by recurrent postprandial hypoglycemia three years after surgery, presented to the hospital with fasting hypoglycemia six months later. She denied family history of insulinoma, pituitary tumor or hyperparathyroidism.

\section{Investigation}

The patient's initial workup was started when she developed symptoms of diaphoresis, dizziness, tremors and syncope after meals. Workup revealed concurrent elevated insulin of $35.5 \mu \mathrm{IU} / \mathrm{mL}$ (normal range $\leq 3 \mu \mathrm{U} / \mathrm{mL}$ ) and C-peptide of $6.75 \mathrm{nmol} / \mathrm{L}$ (normal range $\leq 0.2 \mathrm{nmol} / \mathrm{L}$ ) during a hypoglycemic episode. Serum sulfonylurea screen was negative, insulin antibodies were unremarkable, and serum IGF-2 was within normal limit. To investigate the diagnosis of an insulinoma, a 72-h fast was done revealing stable blood glucose in the $70-80$ 's $\mathrm{mg} / \mathrm{dL}$ range without hypoglycemic events. Imaging studies including CT of the abdomen and pelvis with contrast, MRI of the abdomen and pelvis with contrast, and esophagogastroduadenoscopy with endoscopic ultrasound did not reveal a pancreatic mass. A mixed meal challenge test (MMCT) revealed hyperinsulinemic hypoglycemia after $1 \mathrm{~h}$. Patient was started on a low carbohydrate diet, along with acarbose $50 \mathrm{mg}$ three times daily. One month after initial presentation of symptoms, patient again presented with syncopal episodes due to postprandial hypoglycemia. She underwent revision of her gastric bypass surgery via gastric remnant to gastric pouch anastomosis and jejunal to jejunal anastomosis. Unfortunately, she was only able to tolerate a regular diet for 1 week after the revision and had to be restarted on medical and dietary treatment. She was stabilized on a low carbohydrate diet, diazoxide $75 \mathrm{mg}$ three times daily and octreotide $175 \mu \mathrm{g}$ subcutaneous injection three times daily for a period of 6 weeks, but returned to the hospital with newly developed nighttime, fasting hypoglycemia. Patient complained of arising during the night and in the early morning with hypoglycemic symptoms, with the longest period of fasting being $7 \mathrm{~h}$ after her last meal.

A repeat 72 -h fast study was attempted while the patient was on diazoxide $50 \mathrm{mg}$ three times daily and octreotide $100 \mu \mathrm{g}$ every $8 \mathrm{~h}$. As opposed to the first study, the patient developed hypoglycemia within $7 \mathrm{~h}$ of fasting. Patient had an insulin level of $20 \mu \mathrm{IU} / \mathrm{mL}$ and C-peptide of $3.3 \mathrm{ng} / \mathrm{mL}$ with blood glucose at $41 \mathrm{mg} / \mathrm{dL}$. To rule out other superimposed etiologies of fasting hypoglycemia, namely insulinoma, the patient underwent both a selective arterial calcium stimulation test (SACST) and a 68Ga-DOTATATE PET/CT scan. SACST with hepatic venous sampling resulted in greater than two-fold increase in insulin levels following calcium infusion through the splenic artery as well as superior mesentery artery. A 68Ga-DOTATATE PET/CT showed no evidence of radiotracer-avid neoplasm.

Increasing doses of diazoxide and octreotide were used to control the patient's hypoglycemia but were unsuccessful. Patient ultimately underwent partial pancreatectomy with $80 \%$ resection. The pancreas was extensively serially sectioned and examined and palpated, and no discrete lesions or nodules were identified. In the absence of any discrete lesion, the resection margin and a random sampling of remaining pancreatic tissue was submitted for evaluation, for a total of five sections across the specimen. Histopathology showed pancreatic parenchyma with enlarged islets and cells with large hyperchromatic nuclei, consistent with diffuse nesidioblastosis (see Figs 1,2 and 3 ). Those histopathological findings were present diffusely throughout all islets identified, which would render a single (or multifocal) lesion less likely.

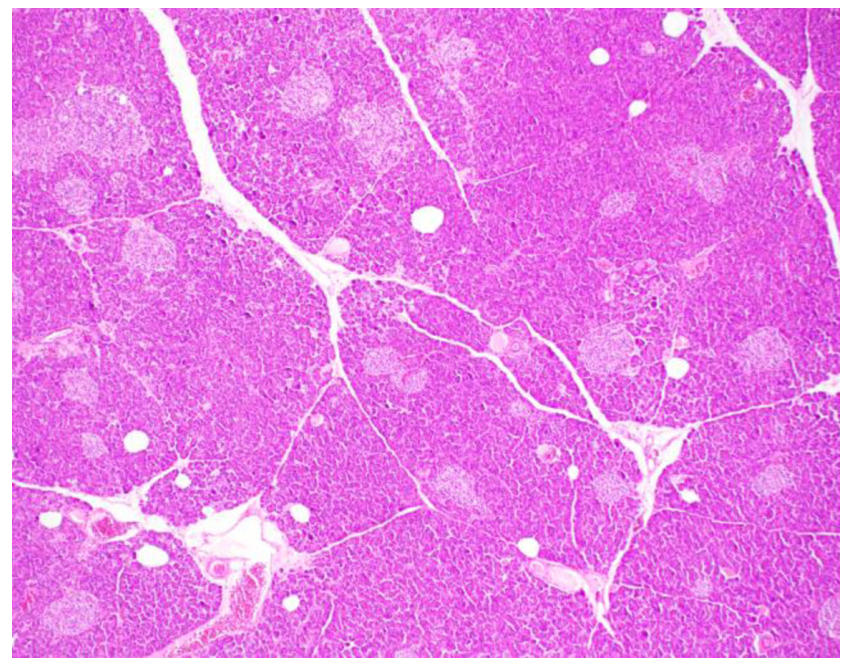

Figure 1

Hematoxylin and eosin-stained sections of the patient's pancreatic tissue, showing increased number of islets with variations in size and shape. 

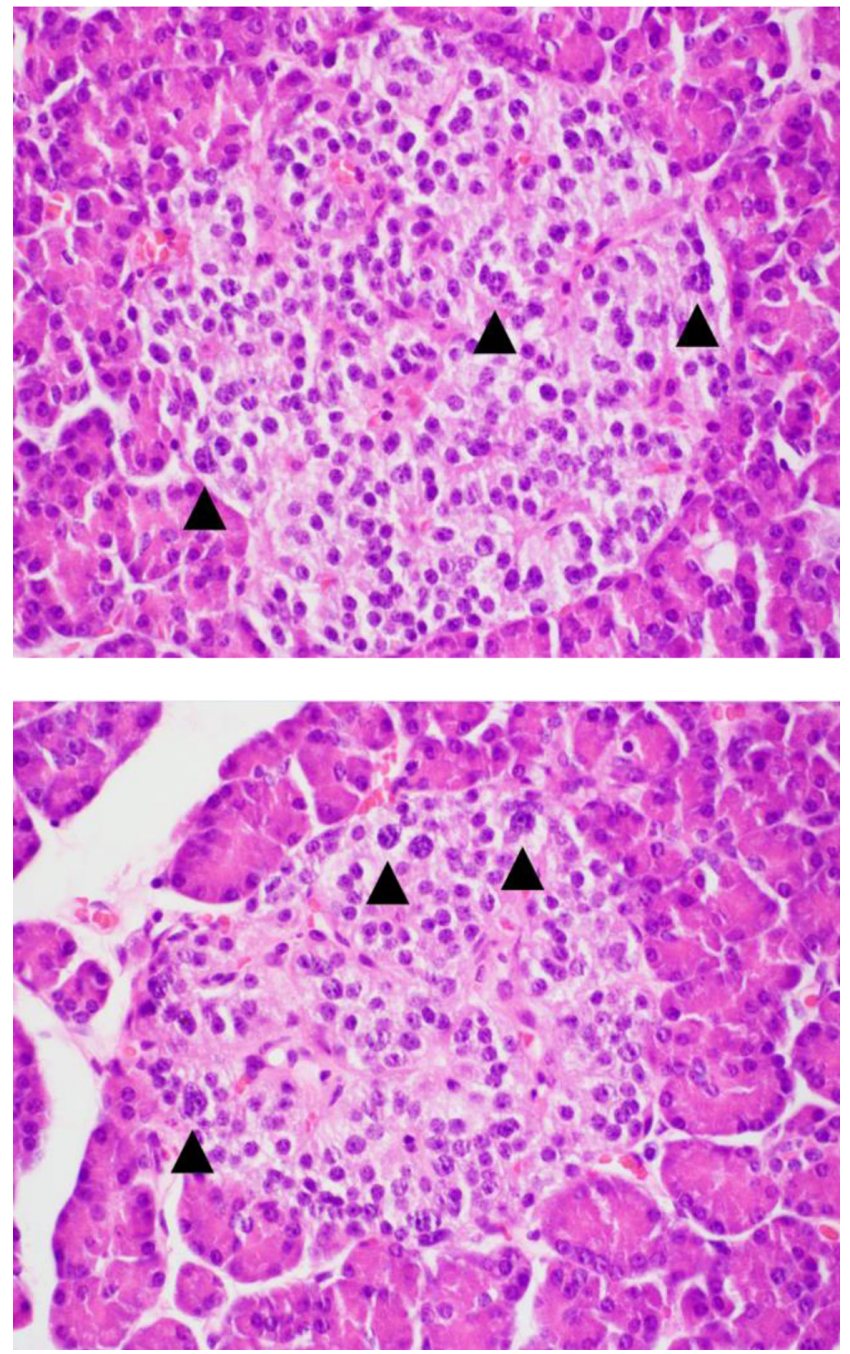

Figure 2

Higher magnification of pancreatic islets. Scattered neuroendocrine cells are seen with enlarged nuclei, irregular nuclear contours, coarse chromatin and variably prominent nucleoli (arrowheads).

\section{Treatment}

Patient was unsuccessfully treated with dietary modifications, medical management, including acarbose $50 \mathrm{mg}$ orally three times daily, diazoxide $75 \mathrm{mg}$ three times a day, octreotide $175 \mathrm{mg}$ SQ three times a day and revision of her gastric bypass. She ultimately underwent pancreatectomy with $80 \%$ resection.

\section{Outcome and follow-up}

Patient is off diazoxide and octreotide and on a restricted carbohydrate diet. Patient had no hypoglycemic episodes at 1-month outpatient follow-up. Thus far, she has not been readmitted to a hospital.
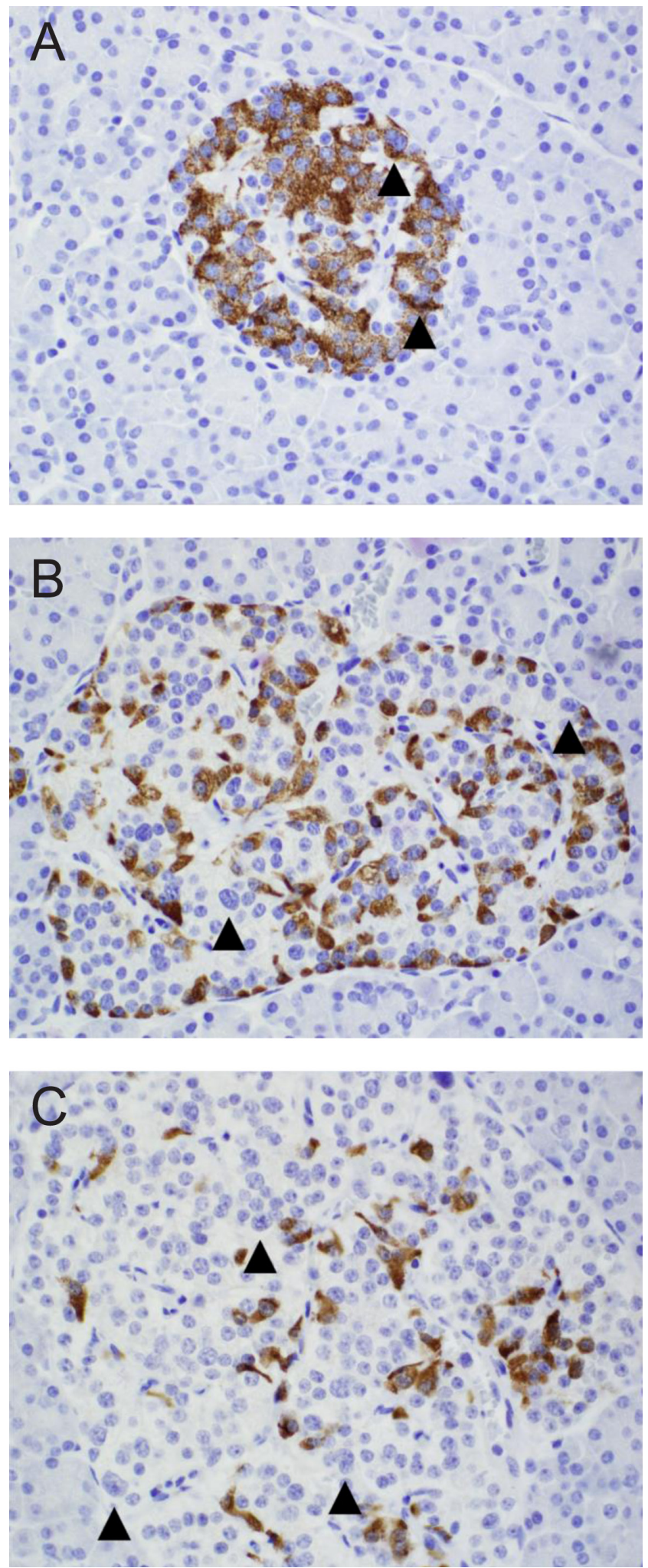

Figure 3

Immunohistochemical staining was performed on the patient's tissue. The cells described in Fig. 2 stain positive for insulin (A) but not glucagon (B) or somatostatin (C) (arrowheads). 


\section{Discussion}

Herein, we present a case of PGBH in a patient who initially presented with postprandial hypoglycemia 3 years after RYGB and 6 months later developed fasting hypoglycemia. In PGBH, plasma insulin levels are inappropriately high despite low blood glucose levels usually after meals. Histopathology from such patients who underwent partial/total pancreatomy identified nesidioblastosis, which is characterized by diffuse pancreatic islet involvement with islet hypertrophy, with or without hyperplasia, and enlarged and hyperchromatic cell nuclei $(1,2,4,5)$. Histopathologic findings, however, can be minimal making the pathologic diagnosis challenging $(6,7)$. The exact mechanism causing hypoglycemia in PGBH remains unclear. The excessive release of incretin hormones, gastric inhibitory polypeptide (GIP) and glucagon-like-peptide-1 (GLP-1) are thought to play an important role in PGBH. GLP-1 is mostly secreted in the gut in response to food, causing a glucose-dependent release of insulin and suppression of glucagon. This response has been shown to be exaggerated in PGBH (8). It has also been suggested that increased GIP and GLP-1 released due to accelerated delivery of carbohydrates directly into jejunum stimulates proliferation of pancreatic $\beta$ cells and thus hyperinsulinemia and postprandial hypoglycemia $(1,2,4,9,10)$. Other proposed mechanisms for PGBH include altered glucose absorption with changes in the gut microbiota, bile acid level and composition (11).

The differential diagnosis of hyperinsulinemic hypoglycemia includes insulinoma, PGBH, late dumping syndrome (LDS) and exogenous causes. Insulinoma is most commonly associated with fasting hypoglycemia and in very rare cases postprandial hypoglycemia (6). In contrast, PGBH and LDS typically present with postprandial hypoglycemia. With LDS, the patient develops dizziness and diaphoresis $1-4 \mathrm{~h}$ after ingestion of a meal (6). LDS is thought to be a result of an early excessive insulin response and subsequent hypoglycemia and is typically not associated with neuroglycopenic symptoms. Some researchers suggest that LDS and PGBH may represent different ends of a spectrum with dumpling syndrome being on one end of spectrum that responds to diet modifications $(4,11,12)$. Of note, large dose of octreotide can potentially aggravate hypoglycemia by inhibiting glucagon secretion, especially in patients with insulinoma. However, we have not found any detailed observation of hypoglycemia induced by octreotide at the typical treatment dose in the literature in patients without neuroendocrine tumor.
A monitored MMTT is the test of choice to diagnose PGBH and should be done prior to 72-h fast and imaging or invasive studies. Only if MMTT is negative, a supervised 72-h fast should be done. The use of SACST has been suggested to localize hyperplastic beta cells to pancreatic arterial distributions in those with PGBH and guide surgical interventions (13). In our patient, a three-fold increase in the relative fold increase in hepatic venous insulin concentration (rHVI) was seen in both splenic artery and superior mesentery artery distributions, suggesting nesidioblastosis as a more likely diagnosis.

PGBH is a challenging complication of gastric bypass. In the most severe cases, patients are refractory to conservative therapy with diet change, medications and reversal of bypass necessitating partial pancreatectomy. Although recurrence is not uncommon after partial pancreatectomy, majority of the patients report improved quality of life and improved symptoms according to a recent study done by Mayo clinic (14). Our patient initially presented with postprandial hypoglycemia after RYGB and later developed fasting hypoglycemia, which is very uncommon in PGBH. Fasting hypoglycemia has been described in sporadic cases of coexisting insulinoma in patients with PGBH (15). To our knowledge, there are only a few case reports of fasting hypoglycemia with PGBH without coexisting insulinoma in the past two decades (3). Our hypothesis for this phenomenon is that this disease is progressive, and later in its course, the insulin release becomes dissociated from food stimulation and is increased at baseline. Therefore, the development of fasting hypoglycemia may be a predictor of more severe refractory disease and likely failure of conservative management. Future studies are needed to investigate the prevalence as well as etiology of progression from postprandial to fasting hypoglycemia.

\section{Declaration of interest}

The authors declare that there is no conflict of interest that could be perceived as prejudicing the impartiality of this case report.

\section{Funding}

This research did not receive any specific grant from any funding agency in the public, commercial or not-for-profit sector.

\section{Patient consent}

Patient consent for the publication of this case report could not be obtained as neither the patient nor a relative could be traced. 


\section{Author contribution statement}

$X$ Chen is a second-year resident in internal medicine who conducted the literature review and manuscript construction. D Kamel is a fellow in endocrinology who provided clinical care for the patient as well as manuscript edition. B Barnett is a consultant endocrinologist who provided clinical care for the patient and assisted in interpretation of imaging studies. E Yung is a consultant pathologist who was responsible for the histology-related aspect of this case and provided clinical care for the patient. A Quinine is a final year medical student who provided patient care. C Nguyen is a consultant endocrinologist who provided clinical care for the patient and was responsible for manuscript construction and advice.

\section{References}

1 Ritz P, Vaurs C, Barigou M \& Hanaire H. Hypoglycaemia after gastric bypass: Mechanisms and treatment. Diabetes, Obesity and Metabolism 201618 217-223. (https://doi.org/10.1111/dom.12592)

2 Cui Y, Elahi D \& Andersen DK. Advances in the etiology and management of hyperinsulinemic hypoglycemia after roux-en-y gastric bypass. Journal of Gastrointestinal Surgery 201115 1879-1888. (https://doi.org/10.1007/s11605-011-1585-8)

3 Taha M, Qintar M \& Sibai F. Hypoglycemia due to an adult-onset nesidioblastosis, a diagnostic and management dilemma. Avicenna Journal of Medicine 20122 45. (https://doi.org/10.4103/22310770.99164)

4 Rariy CM, Rometo D \& Korytkowski M. Post-gastric bypass hypoglycemia. Current Diabetes Reports 201616 19. (https://doi. org/10.1007/s11892-015-0711-5)

5 Ünal B, Uzun ÖC, Başsorgun CI, Erdoğan O \& Elpek GÖ. A rare complication of gastric bypass (weight loss) surgery. International Journal of Surgical Pathology 201423 68-70. (https://doi. org/10.1177/1066896914554833)

6 Millstein R \& Lawler HM. Hypoglycemia after gastric bypass: an emerging complication. Cleveland Clinic Journal of Medicine $2017 \mathbf{8 4}$ 319-328. (https://doi.org/10.3949/ccjm.84a.16064)
7 Klöppel G, Anlauf M, Raffel A, Perren A \& Knoefel WT. Adult diffuse nesidioblastosis: genetically or environmentally induced? Human Pathology 200839 3-8. (https://doi.org/10.1016/j. humpath.2007.09.010)

8 Salehi M, Gastaldelli A \& D’Alessio DA. Blockade of glucagonlike peptide 1 receptor corrects postprandial hypoglycemia after gastric bypass. Gastroenterology 2014146 669-680. (https://doi. org/10.1053/j.gastro.2013.11.044)

9 Abellán P, Cámara R, Merino-Torres JF, Pérez-Lazaro A, del Olmo MI, Ponce JL, Rayón JM \& Piñón F. Severe hypoglycaemia after gastric bypass surgery for morbid obesity. Diabetes Research and Clinical Practice 2008 79 7-9. (https://doi.org/10.1016/j.diabres.2007.07.017)

10 Mingrone $\mathrm{G} \&$ \& Castagneto M. Bariatric surgery: unstressing or boosting the $\beta$-cell? Diabetes, Obesity and Metabolism 200911 130-142. (https://doi.org/10.1111/j.1463-1326.2009.01120.x)

11 Ashrafian H, Athanasiou T, Li JV, Bueter M, Ahmed K, Nagpal K, Holmes E, Darzi A. \& Bloom SR diabetes resolution and hyperinsulinaemia after metabolic Roux-en-Y gastric bypass. Obesity Reviews 201112 257-272. (https://doi.org/10.1111/j.1467789x.2010.00802.x)

12 Pathak R, Karmacharya P, Salman A \& Alweis R. An unusual cause of hypoglycemia in a middle-aged female after bariatric surgery. Journal of Community Hospital Internal Medicine Perspectives 2014423118. (https://doi.org/10.3402/jchimp.v4.23118)

13 Thompson SM, Vella A, Thompson GB, Rumilla KM, Service FJ, Grant CS \& Andrews JC. Selective arterial calcium stimulation with hepatic venous sampling differentiates insulinoma from nesidioblastosis. Journal of Clinical Endocrinology and Metabolism 2015 100 4189-4197. (https://doi.org/10.1210/jc.2015-2404)

14 Vanderveen KA, Grant CS, Thompson GB, Farley DR, Richards ML $\&$ Vella A. Outcomes and quality of life after partial pancreatectomy for noninsulinoma pancreatogenous hypoglycemia from diffuse islet cell disease. Surgery 2010148 1237-1245. (https://doi.org/10.1016/j. surg.2010.09.027)

15 Bright E, Garcea G, Ong S, Madira W, Berry PB \& Dennison AR. An unusual case of concurrent insulinoma and nesidioblastosis. Journal of the Pancreas 20089 649-653.

Received in final form 27 July 2018

Accepted 9 August 2018 